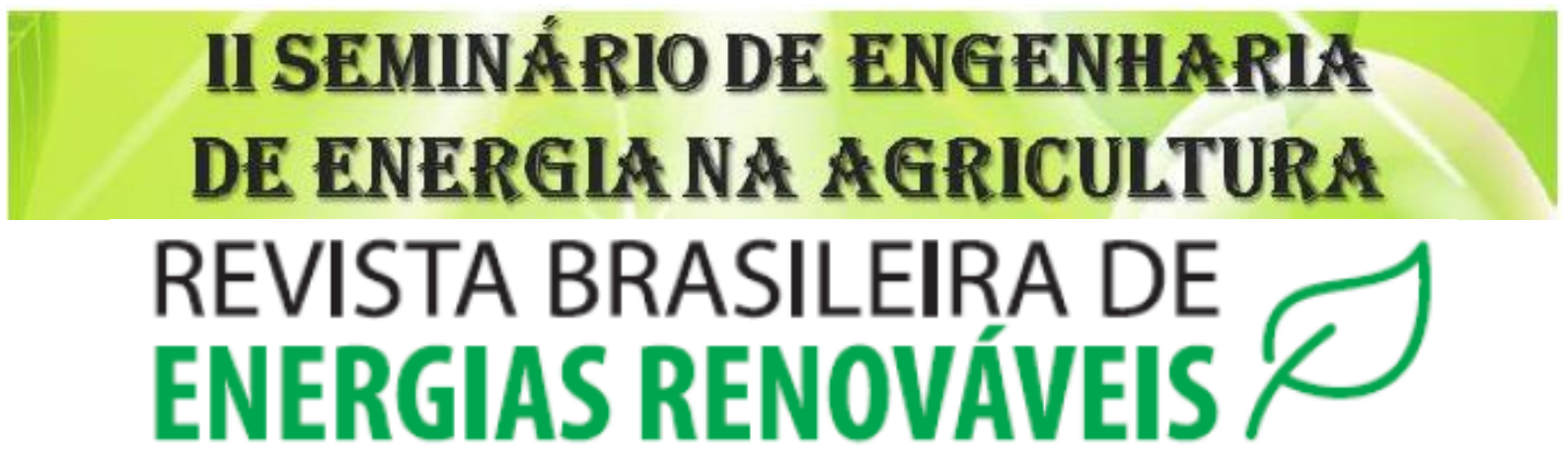

\title{
MÉTODOS DE DETERMINAÇÃO DA RADIAÇÃO SOLAR GLOBAL EM DIFERENTES PLANOS INCLINADOS ${ }^{1}$
}

\author{
Michael Collin Vargas ${ }^{2}$, Jair Antonio Cruz Siqueira ${ }^{3}$, Carlos Eduardo Camargo \\ Nogueira $^{3}$, Marcelo Machado Marquez Zampiva ${ }^{2}$, Eduardo Argou Aires Cunha ${ }^{2}$ Carlos \\ Adriano Bohn ${ }^{3}$
}

\begin{abstract}
${ }^{1}$ Apresentado no $2^{\circ}$ Seminário de Engenharia de Energia na Agricultura: 30/11/2017UNIOESTE, Campus Cascavel.

${ }^{2}$ Universidade Estadual do Oeste do Paraná - UNIOESTE, Curso de Engenharia Civil. Rua Universitária 2069, Jardim Universitário, CEP 85819-110, Cascavel - PR.

michaelcollin95@gmail.com

${ }^{3}$ Universidade Estadual do Oeste do Paraná - UNIOESTE, Programa de Pós-graduação em Engenharia de Energia na Agricultura. Rua Universitária, 2069, CEP: 85.819-110, Jardim Universitário, Cascavel, PR.
\end{abstract}

\section{RESUMO}

Este trabalho teve por objetivo avaliar os métodos utilizados para determinação da radiação solar global em diferentes planos inclinados. Para isso avaliou-se as características apresentadas pelos métodos de: Angström-Prescott, Hargreaves-Samani, Thornton-Running, Weiss, Kondratyev e Manolova, Aragon e Toledo Piza e, Colle e Pereira. Por conseguinte, os modelos matemáticos diferem entre si pelo grau de complexidade e pelas variáveis de entrada. Sendo que o maior diferencial são os ajustes dos coeficientes de determinação das equações.

Palavras-chave: Radiação solar global, radiação solar no plano inclinado, modelos de radiação solar. 


\title{
METHODS OF DETERMINATION OF GLOBAL SOLAR RADIATION IN DIFFERENT INCLINED PLANES
}

\begin{abstract}
The objective of this work was to evaluate the methods used to determinate global solar radiation in planes of different inclinations. For this, it evaluated the characteristics displayed by the following methods: Angström-Prescott, Hargreaves-Samani, Thornton-Running, Weiss, Kondratyev and Manolova, Aragon and Toledo Piza, Colle and Pereira. The main considerations pointed out that the mathematical models differ by level of complexity and input variables. The biggest differential is in the adjustment of the equations determination coefficients.
\end{abstract}

Keyword: Global solar radiation, solar radiation on inclined plane, solar radiation model.

\section{Introdução}

Segundo Santos (2009), a sustentabilidade ambiental é um dos temas mais debatidos na atualidade. Fóruns mundiais debatem frequentemente a utilização dos recursos naturais e sua preservação para gerações futuras, com enfoque também à preocupação com a matriz energética: elemento significativo de degradação ambiental e, ao mesmo tempo, a base da evolução tecnológica humana.

A hidroeletricidade é a principal forma de energia elétrica que supre a demanda existente hoje no Brasil, cerca de $80 \%$. Entretanto causam grande impacto ambiental e social proveniente dos alagamentos nas áreas em que são implantadas. Além disso, o setor energético brasileiro enfrenta também a alta demanda de energia, e consequente insuficiência em sua oferta, gerando crises como os "apagões", em 2001, e problemas frequentes nos horários de pico da demanda (Santos et al., 2009).

Devido a intempéries relacionadas às chuvas e seus regimes, a expansão do consumo de energia no mundo contemporâneo e a utilização cada vez maior de recursos não renováveis do planeta, apresentam-se como grandes desafios a serem enfrentados pela humanidade e que demandam soluções cada vez mais criativas. Tem-se de certa forma uma vulnerabilidade na rede elétrica e que nem sempre consegue atender a demanda nacional (Takenaka, 2010).

Revista Brasileira de Energias Renováveis, v.8, n.4, p.698- 707, 2019 
Destarte, temos as fontes renováveis que têm como vantagem a geração de energia sem danos significativos ao meio ambiente. Entre as novas alternativas para uma produção de energia de maneira sustentável e limpa, destaca-se a geração solar fotovoltaica (FV) por ser uma fonte de conversão direta da energia solar em energia elétrica de maneira não poluente, silenciosa, eficiente e não prejudicial ao meio ambiente (RÜTHER, 2004).

No Brasil, com o advento da resolução 482/2012 da ANEEL (ANEEL, 2012), foi permitida a instalação de sistemas conectados à rede de energia elétrica convencional por meio do sistema de compensação de créditos. Esses sistemas quando utilizados em unidades consumidoras residenciais, são instalados sobre os telhados das residências. Por sua vez, os telhados possuem diversas inclinações de acordo com o tipo de material utilizado para cobertura. Além disso, apresentam orientações com relação a incidência dos raios solares em desacordo com a orientação recomendada para a instalação de sistemas fotovoltaicos. No hemisfério sul, a recomendação é orientar os sistemas fotovoltaicos para o norte verdadeiro e incliná-los nesta direção em um ângulo corresponde à latitude do local. Assim definido visando um melhor aproveitamento da captação da energia solar com base na radiação solar global incidente na superfície.

Assim, discorrer-se-á aqui sobre os vários métodos para obtenção da energia solar global através de suas variabilidades e expô-los de forma mais usual para se ter uma ideia geral de como se estabelece a relação da energia advinda do sol com a que realmente chega na superfície fotovoltaica.

O presente trabalho consiste em uma revisão bibliográfica, sobre cinco métodos utilizados para a determinação da radiação solar global sobre o plano inclinado.

\section{Materiais e métodos}

\section{Radiação solar global}

A radiação solar global incidente sobre uma superfície plana e horizontal é uma fração da radiação solar no topo da atmosfera. Esta fração de radiação que chega à superfície depende das condições atmosféricas locais, as quais caracterizam a transmitância atmosférica para a radiação de ondas curtas como se segue na equação abaixo (BORGES et al., 2010): 


$$
R_{s}=K_{t} \cdot R_{a}
$$

Sendo $R_{s}$ a radiação solar incidente, $K_{t}$ o coeficiente de transmissividade atmosférico adimensional e $R_{a}$ a radiação solar no topo da atmosférica.

Os modelos dispostos aqui baseiam-se na Equação 1, diferindo em relação à determinação do coeficiente de transmissividade atmosférica Kt, além de todos utilizarem a amplitude térmica diária como principal variável de entrada. Os modelos de Thornton-Running e o de Weiss são versões modificadas do modelo de Bristow e Campbell (1984). Isto posto, foram mantidas as constantes originalmente propostas, cujo nesse trabalho não se objetivou a calibração local dos mesmos (BORGES et al., 2010).

Por vezes pode-se não ter dados coletados disponíveis de radiação solar, porquanto recorrer-se-á modelos matemáticos que estimem-na com boa precisão. Primeiramente podemos destacar a equação descrita por Angström - Prescott (MARTÍNEZ-LOZANO et al., 1984), usando os coeficientes "a" e "b" e a radiação extraterrestre.

\section{Modelo de Angström - Prescott}

Para a determinação dos valores da radiação solar global diária horizontal inclinada e, direta na incidência normal, o modelo de Angström-Prescott descrito por Iqbal (1983) é apresentado abaixo. Os dados utilizados no modelo são obtidos por meio da integração das respectivas curvas de radiação instantânea, integrada do nascer ao pôr do sol. A projeção da radiação direta diária na horizontal foi dada pelo produto entre e o cosseno do ângulo zenital diário.

A radiação incidente no topo da atmosfera para uma superfície horizontal $\left\langle H_{0 H}^{d}\right\rangle$ pode ser estimada pela seguinte equação:

$$
\left\langle H_{0 H}^{d}\right\rangle=\left(\frac{24}{\pi}\right) H_{\Xi E} E_{0}\left[\left(\frac{\pi}{180}\right) \omega_{s}(\sin \delta \sin \varphi)+\left(\cos \delta \cos \varphi \sin \omega_{s}\right)\right]
$$

Em que: $H_{s c}$ é conhecida como a constante solar, $E_{0}$ é o fator de correção da excentricidade da órbita da Terra. Segundo Iqbal (1983), o fator de excentricidade pode ser calculado pela equação 3 : 
$E_{0}=1,000110+0,034221 \cos \Gamma+0,001280 \sin \Gamma+0,000719 \cos 2 \Gamma+0,000077 \sin 2 \Gamma$

Em que:

$$
\Gamma=\frac{2 \pi(D J-1)}{366}
$$

Sendo DJ o dia juliano, em dias.

E $\omega_{s}$ é o ângulo horário solar diário horizontal, calculado pela equação:

$$
\omega_{s=\cos ^{-1}}(-\tan \varphi \tan \delta)
$$

E $\omega_{s}^{\prime}$ é o ângulo horário solar diário inclinado, calculado pela equação:

$$
\omega_{s=\min }^{\prime}\left\{\cos ^{-1}(-\tan \varphi \tan \delta) \cos ^{-1}(-\tan \delta \tan \varphi-\beta)\right.
$$

Em que: $\varphi$ é a latitude local; $\beta$ é o ângulo de inclinação; $\delta$ é a declinação solar. Esta última é calculada pela equação:

$$
\delta=23,45 \sin \left[\left(\frac{360}{365}\right)(D J+284)\right]
$$

O fotoperíodo (N) foi obtido de acordo com a seguinte expressão:

$$
N_{=0,133 \cos ^{-1}(-\tan \varphi \tan \delta)}
$$

Obtidos os valores de radiação extraterrestre $\left\langle H_{0 H}^{d}\right\rangle$, e possuindo os valores de $\left\langle H_{g H}^{d}\right\rangle$, sendo estes os valores da radiação solar global diária, para cada dia do ano, agrupam-se os dados e por meio de análises de regressão linear se obtém os valores dos coeficientes "a" e "b" da equação de Angström-Prescott mostrada abaixo.

$$
\left\langle H_{g H}^{d}\right\rangle=\left(a+b \frac{n}{N}\right)\left\langle H_{0 H}^{d}\right\rangle
$$

Em que n é o número de horas de brilho solar e $\mathrm{N}$ é o número máximo de horas de brilho solar, mostrado acima. 
Algumas análises ainda podem serem feitas a partir da obtenção dos coeficientes a e b estimados pelas seguintes metodologias: coeficientes "a" e "b" mensais; coeficientes "a" e "b" anual; coeficientes "a" e "b" geral; coeficientes "a" e "b" propostos por Glover e Mcculloch (1958):

$$
\mathrm{A}=0,29 \cdot \cos (\varphi) \text { e } \mathrm{b}=0,52
$$

A $\left\langle H_{g H}^{d}\right\rangle$ então estimada e correlacionada com os valores de $\left\langle H_{g H}^{d}\right\rangle$ obtidos pela estação convencional por meio de análise de regressão.

Por conseguinte, para superfícies inclinadas, a radiação solar diária incidente no topo da atmosfera para as superfícies horizontal $\left\langle H_{0 H}^{d}\right\rangle$ e inclinada $\left\langle H_{0 \beta}^{d}\right\rangle$ foi estimada segundo Iqbal (1983). Nas superfícies inclinadas a radiação direta diária foi obtida pela aplicação do fator geométrico $\left\langle R_{b}\right\rangle$, que traduz a relação entre a radiação no topo da atmosfera para a superfície inclinada e para a superfície horizontal, mostrada na equação 11 (Simonson, 1981; Iqbal, 1983; Wenxian et al., 1995):

$$
\left\langle H_{\beta 0}^{d}\right\rangle=\left(\frac{24}{\pi}\right) I_{\Omega \varepsilon} E_{0}\left[\left(\frac{\pi}{180}\right) \omega_{s}(\sin \delta \sin (\varphi-\beta))+\left(\cos \delta \cos (\varphi-\beta) \sin \omega_{s}^{\prime}\right)\right]
$$

\section{Modelo de Hargreaves-Samani (HS)}

Para Borges et al. (2010), Hargreaves e Samani (1982) foram uns dos primeiros a propor estimativas de radiação solar incidente a partir da temperatura do ar, por meio da Equação 12:

$$
R_{s}=K_{t^{*}} \cdot\left(T_{x}-T_{m}\right)^{0,5} \cdot R_{n}
$$

Sendo $K_{t}$ o coeficiente de calibração; $T_{x}$ a temperatura máxima do ar; $T_{n}$ a temperatura mínima do ar e, $R_{a}$ sendo a radiação solar no topo da atmosfera.

\section{Modelo de Thornton-Running (TR)}

Para Borges et al. (2010), o modelo de Thornton-Running, utiliza a amplitude térmica diária e mensal para determinação do coeficiente de transmissividade atmosférica, sendo que a Radiação solar na superfície do plano inclinado é dada pela equação 13:

$$
R_{x}=R_{x n}\left[1-0,9 \exp \left(-B\left(T_{x}-T_{n}\right)^{1,5}\right)\right]
$$


$\mathrm{Na}$ equação 11, B é um coeficiente empírico de ajuste adimensional. O mesmo é determinado com base nas normais climatológicas do local através da amplitude térmica mensal média.

\section{Modelo de Weiss (WS)}

Para Borges et al. (2010), este é um dos modelos mais simplórios avaliados por Weiss et al. (2001). Utiliza, além da amplitude térmica do ar, a radiação solar no topo da atmosfera para estimativa de Kt. Segue a equação 14, abaixo, tendo em vista que todos os coeficientes já foram definidos anteriormente:

$$
R_{s}=0,75\left[1-\exp \left(-0,226 \frac{(\Delta T)^{\mathrm{I}}}{R_{n}}\right)\right] \cdot R_{a}
$$

Na equação acima, a amplitude térmica mensal $\Delta T$ é calculada como:

$$
\Delta T=T_{x i}-\left(\frac{T_{n([)}+T_{n(i+1)}}{2}\right)
$$

Com $T_{x x}$ sendo a temperatura máxima do ar; $T_{n}$ a temperatura mínima e i um subscrito indicativo referente ao dia atual.

Por conseguinte, segundo Turco (2006), ainda podemos citar o modelo matemático proposto por Kondratyev, que leva em consideração diferentes exposições e declividades, partindo de determinações na horizontal. Kondratyev e Manolova (1960) estimaram as variáveis do balanço de radiação para 37 direções e ângulos de 15,40 e $65^{\circ}$ (vertical), a cada $30^{\circ}$ de azimute e no zênite, sob céu limpo e nublado na Criméia $\left(45^{\circ} \mathrm{N}\right)$. Usando modelos teóricos e dados dos piranômetros instalados em teodolitos verificaram que, para inclinações até $10^{\circ}$ (menores ou iguais à 17,6\%), a orientação tem pouca influência na variação da radiação total. Todavia, acima de $10^{\circ}$, ela é intrinsicamente dependente da orientação e declividade. Destarte, concluíram que a radiação direta é a principal componente diferencial de orientações e declividades. 
Latanze et al. (1987), utilizando-se do modelo de Kondratyev verificaram que os totais de radiação solar medidos em diferentes orientações e épocas do ano, resultaram em menos de $2 \%$ de diferença em comparação com os valores estimados pelo modelo citado acima.

Para Turco (2006), Aragon e Toledo Piza (1991) formularam um modelo teórico para o cálculo da energia solar total diária incidente em rampas planas com diferentes inclinações e exposições, obtendo-se resultados satisfatórios no contraste entre os valores teóricos e experimentais.

Ainda segundo Turco (2006), Colle e Pereira (1998) foram membros de uma equipe que desenvolveu um modelo computacional denominado BRAZILSR, que determinava a intensidade da radiação solar incidente na superfície a partir de imagens de satélite. Fora validado cotejandose as estimativas do modelo com dados de piranômetros instalados em várias localidades do Brasil.

\section{Considerações Finais}

Considera-se, que os modelos estudados aqui são necessários para estimar a radiação solar global nas superfícies. Descrita suas metodologias e demonstrando-as, vê-se que uma das principais conclusões a se fazer é quanto às suas diferenças entre si pelo grau de complexidade e pelas variáveis de entrada. Sendo essa última a amplitude térmica diária como uma das principais variáveis de entrada. Assim, a avaliação dos modelos fora feita, como dito anteriormente, mantendo as constantes originais dos modelos e a não calibração local dos mesmos.

Considera-se, ainda, que a radiação direta é a principal componente que é afetada pelas orientações e declividades. Evidencia-se que, inclinações em até $10^{\circ}$ apresentam baixa influência na variação da radiação solar global estimada.

Considera-se, por fim, que os totais de radiação solar estimados em diferentes orientações e épocas do ano, podem resultar em até menos de $2 \%$ de diferença em comparação com os valores estimados.

\section{Referências}

ARAGON, F.F.; TOLEDO PIZA, J.A.N. Modelo matemático generalizado de radiação solar

diária sobre superfícies planas. Energia na Agricultura, Botucatu, v.6, n.1, p.5-14, 199 
BORGES, V.P. et al. Avaliação de modelos de estimativa da radiação solar incidente em

Cruz das Almas, Bahia. Revista Brasileira de Engenharia Agrícola e Ambiental. Campina Grande, PB, UAEA/UFCG, v.14, n.1, p.74-80, 2010.

COLLE, S; PEREIRA, E.B. Atlas de irradiação solar do Brasil. Brasília: LABSOLAR, 1998. $65 \mathrm{p}$.

HARGREAVES, G. H.; Samani, Z. A. Estimating potential evapotranspiration. Journal of Irrigation and Drainage Engineering, v.108, p.225-230, 1982.

KONDRATYEV, K.Y. Radiation regime of inclined surfaces. Genebra; World Meteorological Organization, $1977.82 \mathrm{p}$.

KONDRATYEV, K.Y.; MANOLOVA, M.P. The radiation balance of slopes. Solar Energy, Kidlinton, v.4, n.1, p.14-19, 1960.

LATANZE, R.J.; BENINCASA, M.; LOPES, L.R. Estimativa da radiação solar incidente sobre superfícies com diferentes orientações. CONGRESSO BRASILEIRO DE ENGENHARIA AGRÍCOLA, Jundiaí, p.646-7, 1987.

RÜTHER, R. Edifícios Solares Fotovoltaicos: O Potencial da Geração Solar Fotovoltaica Integrada a Edificações Urbanas e Interligada à Rede Elétrica Pública. Editora da UFSC. LABSOLAR. Florianópolis, 2004.

RÜTHER, R.; REGUSE, W.; DACOREGIO, M. M.; RICARDO, R. V.; JARDIM, C.; PARECY, E. Avaliação do Impacto da Geração Distribuída Utilizando Sistemas Solares Fotovoltaicos Interligados à Rede de Distribuição. In: Seminário de pesquisa e desenvolvimento - CELESC, Anais. Florianópolis - SC, 2004.

SANTOS, I. P. Integração de painéis solares fotovoltaicos em edificações residenciais e sua contribuição em um alimentador de energia de zona urbana mista. Universidade Federal de 
Santa Catarina - UFSC, Programa de pós-gradução em engenharia civil - PPGEC. Florianópolis - SC, fevereiro de 2009.

SIQUEIRA, J. A. C. Desempenho de um sistema híbrido eólico-fotovoltaico de pequeno porte para energização rural. Tese (Doutorado Agronomia - Área de Concentração em Energia na Agricultura). Universidade Estadual Paulista "Júlio de Mesquita Filho", Faculdade de ciências agronômicas Campus de Botucatu. Botucatu - SP, dezembro de 2005.

TAKENAKA, F. O. Avaliação do potencial de geração de energia solar fotovoltaica na cobertura das edificações do campus i - cefet- mg, interligado à rede elétrica. Dissertação (Mestrado Engenharia Civil). Universidade Federal de Santa Catarina, programa de pósgraduação em engenharia civil, Minter. Florianópolis - SC, julho de 2010.

TURCO, J. E. P.; RIZZATTI, G.S. Avaliação de modelo matemático para estimar a radiação solar incidente sobre superfícies com diferentes exposições e declividades. Artigo técnico. Departamento de Engenharia Rural da UNESP, Câmpus de Jaboticabal - SP, v.26, n.1, p.257267, jan./abr. 2006.

WEISS, A.; HAYS, C. J. Simulation of daily solar irradiance. Agricultural and Forest Meteorology, v.123, n.3-4, p.187-199, 2004.

WEISS, A.; HAYS, C. J.; HU, Q.; EASTERLING, W. E. Incorporating bias error in calculating solar irradiance: implications for crop simulations. Agronomy Journal, v.93, n.6, p.1321-1326, 2001. 